\title{
Redécouverte d'une grotte de Salomon de Caus à Heidelberg ?
}

La Brunnenstube au Hortus Palatinus

Rediscovery of a grotto of Salomon de Caus in Heidelberg? The Brunnenstube at Hortus Palatinus

\section{Sigrid Gensichen}

\section{(2) OpenEdition}

\section{Journals}

\section{Édition électronique}

URL : http://journals.openedition.org/artefact/5573

DOI : 10.4000/artefact.5573

ISSN : 2606-9245

\section{Éditeur :}

Association Artefact. Techniques histoire et sciences humaines, Presses universitaires du Midi

\section{Édition imprimée}

Date de publication : 15 juillet 2020

Pagination : 129-147

ISBN : 978-2-8107-0691-4

ISSN : 2273-0753

\section{Référence électronique}

Sigrid Gensichen, «Redécouverte d'une grotte de Salomon de Caus à Heidelberg ? », Artefact [En ligne] 12 | 2020, mis en ligne le 21 décembre 2020, consulté le 23 décembre 2020. URL : http:// journals.openedition.org/artefact/5573; DOI : https://doi.org/10.4000/artefact.5573

\section{$@ \oplus \Theta \Theta$}

Artefact, Techniques, histoire et sciences humaines est mise à disposition selon les termes de la Licence Creative Commons Attribution - Pas d'Utilisation Commerciale - Pas de Modification 4.0 International. 


\title{
Redécouverte d'une grotte de Salomon de Caus à Heidelberg ?
}

\author{
La Brunnenstube au Hortus Palatinus
}

\section{Sigrid Gensichen}

\section{Résumé}

Les jardins du château de Heidelberg, le Hortus Palatinus, possèdent une petite salle voûtée très peu connue, la «Brunnenstube », située dans un mur de soutènement des terrasses. Quelques fragments d'une décoration de grotte y sont conservés : ils ont pu être documentés dans les archives afin de déterminer la fonction de cette salle. Ces vestiges, ainsi qu'une statue de Nymphe récemment retrouvée, datent de l'origine du Hortus Palatinus et peuvent donc être attribués à Salomon de Caus, bien que la salle n'apparaisse pas dans sa description du jardin publiée en 1620. Ainsi, l'analyse de ces fragments de la Brunnenstube apporte des observations originales sur la décoration des grottes de Salomon de Caus à Heidelberg. Elle contribue à une approche archéologique dans la connaissance de ce jardin et des techniques hydrauliques. En effet, la question se pose de la fonction originelle de la Brunnenstube comme réservoir du réseau hydraulique de l'ancien Hortus Palatinus.

\section{Mots-clés}

jardin, Renaissance, fontaines, décoration, château de Heidelberg, Hortus Palatinus, Salomon de Caus

95 Sigrid Gensichen, « Redécouverte d'une grotte de Salomon de Caus à Heidelberg ? La Brunnenstube au Hortus Palatinus », Artefact, 12, 2020, p. 129-147. 


\section{Rediscovery of a grotto of Salomon de Caus in Heidelberg? The Brunnenstube at Hortus Palatinus}

\section{Abstract}

The gardens of Heidelberg Castle, the Hortus Palatinus, contain a small, little-known vaulted hall, the "Brunnenstube", located in a retaining wall of the terraces. Some fragments of a grotto decoration are preserved there: they have been documented in the archives to determine the function of this room. These remains, as well as a recently found statue of a Nymph, date from the origin of the Hortus Palatinus and can therefore be attributed to Salomon de Caus, although the room does not appear in his description of the garden published in 1620. The analysis of these fragments of the Brunnenstube provides some interesting observations on the decoration of the Salomon de Caus grottos in Heidelberg. It contributes to an archeological approach in the knowledge of the gardens history and its hydraulic techniques. Indeed, the question arises as to the original function of the Brunnenstube as a reservoir for the hydraulic network of the former Hortus Palatinus.

\section{Keywords}

garden, Renaissance, fountains, decoration, Heidelberg castle, Hortus Palatinus, Salomon de Caus 


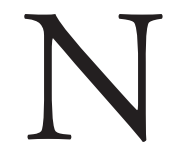

ous voudrions présenter les éléments décoratifs d'une grotte qui ont été retrouvés dans une discrète petite salle voûtée, la Brunnenstube, ainsi qu'un morceau de sculpture figurant une déesse de rivière ou une nymphe de fontaine récemment découvert dans les anciens jardins du château de Heidelberg, le Hortus Palatinus, et que nous proposons d'attribuer à la période de construction réalisée par Salomon de Caus (1576-1626) ou peu de temps après ${ }^{1}$. Cette redécouverte est aussi l'occasion d'une réflexion sur l'alimentation en eau des jardins du côté est de cette résidence princière, qui avait déjà une grande renommée en son temps. C'est le prince-électeur du Palatinat Frédéric V (1596-1632) qui commanda ce gigantesque chantier, immédiatement après son mariage en 1613 avec Élisabeth Stuart (1596-1662), la fille du roi d'Angleterre Jacques $I^{\text {er }}$. Salomon de Caus avait rejoint son nouvel employeur depuis l'Angleterre. Précédemment, il avait travaillé pour la cour de Bruxelles dans le parc de la Warande du palais de Coudenberg, à partir de 1597 jusqu'en 1610, entre autres pour le prince héritier Henri-Frédéric, le frère de la future épouse anglaise du prince-électeur ${ }^{2}$.

Les quelques vestiges qui subsistent sont particulièrement précieux car aucun autre décor créé par Salomon de Caus n'a été matériellement conservé parmi tous les ouvrages qui lui sont attribués avec certitude. Seule la découverte, publiée en 1991 et développée en 2014, de nombreux éléments d'une grotte dans le château de Dieppe est associée à Salomon de Caus ${ }^{3}-$ il appartenait à une famille de Dieppe ${ }^{4}$. Le Hortus Palatinus est documenté par plusieurs sources écrites et graphiques ${ }^{5}$, tandis que la structure architecturale des terrasses, avec des murs de soutènement et des salles souterraines vides ont été conservées. Toutefois, les destructions des

\footnotetext{
1. Cette étude complète mon texte sur le Hortus Palatinus, en allemand, dans lequel toutes les références et les détails de l'iconographie sont donnés : Gensichen, 2019.

2. Sur les ouvrages de Salomon de Caus à Bruxelles : De Jonge, 2000, p. 89-105; Devigne, 1936.

3. Ickowicz, 1991 ; Ickowicz, 2014, p. 80-81 ; Ickowicz, 2017. Je remercie Pierre Ickowicz, conservateur en chef du château-musée de Dieppe, de m’avoir contactée et de m’avoir informée des dernières découvertes.

4. Vérin, 2017, p. 67 et notes 7, 8 et 10.

5. Surtout les gravures de De Caus, 1620, la Scenographia de Matthäus Merian, vers 1620, et le grand tableau de Jacques Fouquier, avant 1620, Kurpfälzisches Museum Heidelberg. Pour les sources écrites plus récentes, c'est-à-dire les livraisons importantes de sable de 1616, le contrat du maître des cavernes (Grottenmeister) de 1619, un "Verzeichnis der paum... " de 1635, plusieurs récits de voyage, etc. Voir les textes dans Hepp, 2008 et Gensichen, 2019, p. 132, n. 6. Plusieurs références plus récentes sur les sources aussi dans Vérin, 2017.
} 
guerres, le délabrement, les modifications, l'extraction de matériaux, les reconstructions et le désintérêt archéologique jusqu'au $\mathrm{xx}^{\mathrm{e}}$ siècle ont largement fait disparaitre les équipements techniques, sculpturaux et décoratifs. L'inventaire de ce qui subsiste est assez limité : un passage souterrain vers la fontaine du Rocher située à l'extrémité nord de la terrasse du Scheffel ${ }^{6}$, mis au jour lors de travaux, ainsi qu'un fragment sculpté d'un dieu-fleuve se trouvant dans la partie basse de la fontaine du Prince ${ }^{7}$, et désormais aussi le fragment sculpté de la Nymphe des eaux et l'ancienne Brunnenstube évoqués précédemment ${ }^{8}$.

\section{Les fragments sculptés : Dieu-fleuve et Nymphe des eaux}

Dans ses gravures de l'Hortus Palatinus (1620), Salomon de Caus a représenté trois personnifications de rivières : le Rhin, d'après des modèles antiques, le Neckar et le Main'. La statue du Rhin, aujourd'hui remplacée par une copie moderne, était placée sur une île rocheuse dans un bassin sur la terrasse principale devant la grande Grotte. Les feuilles de vigne sur sa tête et autour de ses hanches font référence à des modèles flamands, par exemple un groupe en bronze représentant les dieux planétaires, réalisé par le sculpteur et médailleur Jacques Jonghelinck (1530-1606). Les figures de Saturne et de Mercure ont des rinceaux semblables à celles de la sculpture de Heidelberg. Salomon de Caus a pu avoir connaissance de ces sculptures par l'intermédiaire des gravures de Philipp Galle (1537-1612) ${ }^{10}$. Grâce aux feuilles de vigne, les contemporains pouvaient facilement identifier cette statue comme étant la personnification du fleuve fameux pour ces vignobles, le plus important du comté palatin du Rhin auquel il donnait son nom et autour duquel son territoire s'étendait ${ }^{11}$. Les statues des rivières du Neckar et du Main n'ont été conservées que par un dessin figurant sur la planche $\mathrm{n}^{\circ} 15$ des gravures de Salomon de Caus ; elles devaient être

6. Untermann et Gross, 2005, p. 261-264.

7. Zimmermann, 1980, p. 29 (attribué à Salomon de Caus).

8. Pour les constatations matérielles et techniques : Böttcher, 2019, p. 151-152. Ce document n’a jusqu’à présent été mentionné que par Zimmermann, 1980, p. 32.

9. De Caus, 1620 (édition française, non paginée), Av Lectevr [n 14 et 22], pl. 15 et 24.

10. Sur le groupe de sculptures : Buchanan, 1990 ; Meijer, 1979 ; $c f$. Galle, 1586, RHENVS, pl. 10. 11. Sur la place du Rhin dans le Palatinat: Gensichen, 2019, p. 147-148, n. 59. 
placées au bord d'un bassin qui n’a jamais été réalisé sur la terrasse inférieure $^{12}$, et donc subordonnées au fleuve principal du territoire. De même, Salomon de Caus décrit les trois figures portant des cruches sur les plaques en relief destinées à la grotte nommée la petite sur la terrasse sud la plus élevée, derrière la Grande Galerie : "trois fleuues, qui iettent l'eau par des cruches, \& tombe par des rochers iusques en bas. Lesdites figures doibuent estre couvertes de coquilles $\&$ petites rochers en façon de Mosaique ${ }^{13}$ ".

Un dessin préparatoire de Salomon de Caus pour la planche $\mathrm{n}^{\circ} 26$ de l'Hortus Palatinus montre la disposition prévue ${ }^{14}$. Le jet d'eau sortant de la cruche du Fleuve debout placé contre le mur de gauche tombe sur une plaque en dessous et rejoint la cascade qui coule sur toute la largeur du mur ${ }^{15}$. Un fragment du relief de cette statue a été conservé (mais pas in situ), preuve supplémentaire que les travaux de construction de cette terrasse supérieure avaient continué jusqu'à l'achèvement de certaines parties situées à l'intérieur ${ }^{16}$. En 1767, cette statue a été placée au centre d'une pseudo-grotte au-dessus de l'exutoire de la fontaine du Prince située en contrebas dans le fossé sud ${ }^{17}$.

La Nymphe des eaux, tenant une cruche assise sur un dauphin, représentée sur le plus bas des trois reliefs de la planche $\mathrm{n}^{\circ} 27$ du recueil, est également partiellement conservée (Fig. XXI, cahier couleur). Ce fragment sculpté qui avait échappé jusqu’à présent à la recherche, faisait aussi partie à l'origine de la décoration de la grotte nommée la petite, où il avait été (ou devait être) placé en pendant du dieu-fleuve placé debout sur le mur opposé ou au-dessus de l'entrée. Le fragment de figure, dont l'épiderme est endommagé, a probablement été muré dans le côté est de la salle mitoyenne au nord de la grande Grotte dans les années 1930, à une hauteur d'environ

12. Diener, 2007.

13. De Caus, 1620, non paginé, Av Lectevr [n 27] et pl. 27.

14. Stiftung Preussischer Kulturbesitz Berlin, Kupferstichkabinett, Inv.-Nr. KdZ 25974 (dossier WGi 6/5) : encre et lavis gris et brun sur papier, $243 \times 286 \mathrm{~mm}$. Sur la pochette, une inscription "Salomon de Caus » et au verso «Die kleine Grotte im Churfürstlichen Lustgarten zu Haydelberg » [La petite grotte du jardin de plaisance du prince-électeur à Heidelberg] ; Gensichen, 2019, p. 134, n. 17.

15. De Caus, 1620, pl. 26.

16. Metzger, 2008, p. 67.

17. Zimmermann, 1980, p. 29. 
1,50 mètre $^{18}$. Sa posture et son physique étaient également similaires à l'une des figures gravées par Philipp Galle, la nymphe Lynope ${ }^{19}$.

\section{La Brunnenstube : une grotte dans le mur de soutènement sud}

L'emplacement de la salle dans le plus haut mur de soutènement du jardin ${ }^{20}$ suggère qu’il a été déterminé par le système de distribution de l'eau du palais et des jardins, sur lequel il est nécessaire de s'arrêter un instant. Il est évident que le plan du site de l'Hortus Palatinus diffère des résidences tels que Pratolino ou Saint-Germain-en-Laye, principalement en raison de l'absence d'axe central. La position décentrée des jardins, aménagés à l'est du château et coincés entre les coteaux de la montagne et la ville longeant le Neckar, les rapproche plus des jardins de la Warande - bâtis quelques années plus tôt sur un terrain beaucoup moins escarpé - au château de Coudenberg pour la cour archiducale de Bruxelles où Salomon de Caus avait travaillé. Les terrasses en forme de $\mathrm{L}$ du jardin de l'électeur palatin ont dû être aménagées en partie dans une vallée profonde sur le versant nord de la montagne nommée Königstuhl dont la partie supérieure a dû être partiellement remblayée et nivelée avec beaucoup d'efforts.

Ces travaux difficiles ont néanmoins permis d'obtenir un emplacement avantageux : grâce à une situation géologique particulière - des couches de grès aquifères reposent sur du granit imperméable - les sources affleurant sur les coteaux étaient assez nombreuses pour alimenter le château et la ville, chacune avec leur puits ${ }^{21}$. En 1550, le prince-électeur du Palatinat, Frédéric II du Palatinat (1482-1556) ${ }^{22}$, fit capter une première source sur le coteau de Schlierbach, dans le parc de chasse situé à l'est du château. La construction d'un réservoir (transformé plus tard en vivier), d'un puisard

18. Gensichen, 2019, p. 134.

19. Galle, 1587 , pl. 14.

20. Pour les sources relatives à l'histoire de la construction du mur de clôture : Gensichen, 2010. Les travaux de sécurisation et l'étude du bâti ont été commandés par le Landesbetrieb Vermögen und Bau Baden-Württemberg, Amt Mannheim und Heidelberg : Böttcher, 2011. Les travaux ont été réalisés en 2014 : Gensichen, 2014.

21. En 1772 la ville a dénombré environ cinquante sources sur les coteaux : Gensichen, 2019, p. 135 , n. 19.

22. Il fut électeur à partir de 1544 . 
et d'un petit pavillon de chasse dans que ce lieu de plaisance, connu depuis sous le nom de Fontaine du Loup, témoigne déjà de l'intérêt du prince-électeur pour ces sources de la montagne ${ }^{23}$.

Dans le château, il ne reste que quelques vestiges du vaste réseau hydraulique de captage de sources, de puits et de canalisations qui fut considérablement développé entre le $\mathrm{Xv}^{\mathrm{e}}$ et le $\mathrm{XVII}{ }^{\mathrm{e}}$ siècle. Les circuits d'alimentation étaient particulièrement courts parce que les écoulements profitaient de la pression donnée par la forte déclivité du terrain des côtés sud et est. Il y avait même une source qui remplissait directement le fossé sud. Avant la construction de l'Hortus Palatinus, plusieurs rigoles conduisaient déjà les eaux de la montagne pour l'alimentation du château à partir des coteaux de l'est. Dans la première moitié du XvI ${ }^{\mathrm{e}}$ siècle, l'eau était conduite jusqu'à l'étage supérieur d'une casemate qui, en cas d'attaque, bloquait la douve sud et ensuite l'emplacement du château. Ces anciennes conduites d'alimentation ont été soigneusement préservées lors de la construction de la terrasse principale de l'Hortus Palatinus en les protégeant probablement par plusieurs galeries, dont certaines furent encore accessibles jusqu'aux années 1820, afin que l'eau puisse continuer à être conduite dans le château - désormais situé en dessous de la terrasse principale du nouveau jardin. Contrairement, par exemple, à l'approvisionnement en eau des jardins de la Warande à Bruxelles, du parc de la Villa d'Este ou des installations de Saint-Germain-en-Laye nécessitant des moulins, des pompes, des châteaux d'eau, de longues conduites ou des aqueducs ${ }^{24}$, les courtes distances ont permis de limiter les dépenses en machines et en travaux de construction pour l'approvisionnement en eau de l'Hortus Palatinus. Ces courtes distances ont également favorisé la pureté de l'eau.

L'aménagement de la grotte et du bassin de la Brunnenstube a également bénéficié de la situation de plusieurs sources sur les coteaux avoisinants afin de conduire l'eau par gravitation. Dans les gravures de Salomon de Caus, le plan d'ensemble intitulé Ignografia montre clairement les principales sources de l'approvisionnement en eau (Fig. XXII, cahier couleur). La partie des jardins comprenant les équipements hydrauliques les plus nombreux s'étend du côté sud à la limite est du jardin, en passant par la terrasse

23. Gensichen, 2019, p. 135, n. 20.

24. Pour la Warande : De Jonge, 2000, p. 90 ; pour Saint-Germain-en-Laye : Buffa, Rostaing et Lurin, 2010, p. 104-107. 
intermédiaire supérieure jusqu'à l'extension de la terrasse principale qui se trouve plus au nord. Les bassins de cette terrasse, ainsi que le parterre d'eau avec ses fontaines, recevaient l'eau de la montagne et en plus l'exutoire de la grande Grotte, dont les jeux d'eau créés par De Caus prenaient l'eau d'un réservoir aménagé en bassin de Vénus au-dessus de la grotte. À l'extrémité de cette longue et étroite terrasse où se trouvait la fontaine du Rocher, il y avait une autre conduite venant de la fontaine du Loup ${ }^{25}$. Le second lieu important de l'approvisionnement en eau était la terrasse des bains, vers l'ouest, avec l'aménagement de nombreuses pièces d'eau.

La salle souterraine qui nous intéresse ici se situe approximativement entre ces deux principales sources de l'approvisionnement en eau. Des sources écrites et des plans plus récents indiquent que ce devait être l'une des quatre "Bronnen stuben" mentionnées dans le marché de construction de la grotte en $1619^{26}$ : ainsi, un plan dressé en 1810 (Fig. XXII, cahier couleur) pour examiner un dégât des eaux montre à la fois cette grotte et son alimentation en eau depuis les coteaux au-delà du jardin. La source était située là où se trouve aujourd'hui un réservoir construit en 1897. À partir de cet endroit, une canalisation se dirige environ vers le nord jusqu'à cette salle située dans le mur de soutènement. La source qui avait été captée sur les coteaux lors de la période de construction en 1614-1619 se trouvait probablement déjà à cet endroit ${ }^{27}$.

Toutefois, la salle n'est pas représentée dans l'Ignografia. Sur la vue de l'Hortus Palatinus de Jacques Fouquier et sur la Scenographia de Matthäus Merian (1620), son entrée est difficilement reconnaissable. Johann Metzger, qui s'occupa des jardins du château, l'indique au n ${ }^{\circ} 102$ de son plan d'ensemble publié en 1829. Il mentionne un regard de distribution recueillant l'eau de la montagne qui était ensuite conduite jusqu'aux trois bassins rectangulaires de la terrasse principale par un tuyau (recevant aussi l'exutoire des bains), ce qui confirme le plan de $1810^{28}$. Cette salle peut donc être identifiée probablement comme l'un des ouvrages mentionnés dans

25. Metzger J., 1829, p. 94 ; à propos des travaux de Johann Metzger dans les jardins du château d'Heidelberg: Gensichen et Metzger W., 2008, p. 12-17.

26. Gensichen, 2019, p. 136, n 25 ; GLA KA, 67/930, fol. 544v-545v ; Hubach, 2008, p. 42 ; Metzger W., 2008, p. 66, nº 7 .

27. GLA KA, 391/14863, fol. 9-31 (dessin à la plume réhaussé de gouache bleu et rouge clair, datée de 1810).

28. Metzger J., 1829, p. 69 et 81. 
le marché de construction de la grotte, dénommé réservoir ou cuvette, à partir duquel l'eau était distribuée dans le jardin.

Le rapport d'expertise de 1810 indique aussi le possible débit originel des eaux dans le jardin. Il précise que l'eau de cette salle s'écoulait par un conduit en brique de 45 centimètres de haut vers l'est jusqu'à un escalier en colimaçon carré, qui se trouve également dans le mur, puisqu'elle bifurquait à angle droit dans une pierrée sous les terrasses et sortait par le mur de la terrasse principale. En 1810, un grand jet d'eau était encore produit grâce à la forte déclivité. Il s'élevait au-delà de la terrasse exactement là où se trouvait autrefois le plus au sud des trois bassins accolés au mur de la terrasse principale (il est désormais reconstruit). C'est ensuite que, d'après les gravures, devait se trouver la pièce d'eau avec cinq sculptures. Les eaux de la Brunnenstube alimentaient donc principalement, avec l'exutoire de la grande Grotte, cet axe des bassins d'eaux en orientation nord-sud du côté est du jardin.

Le dégât des eaux intervenu en 1810 sur le mur sud et dans la Brunnenstube a été dévastateur. La paroi sud du mur de la salle s'était effondrée et ses murs latéraux avaient été gravement endommagés : ils ont été consolidés et la paroi sud a été rénovée ${ }^{29}$. De surcroît, il manque des sources écrites plus anciennes sur cette salle - de Caus n'en parle pas. En outre, il ne reste que quelques vestiges des grottes. Le jardinier du château, Peter Leonhardt, rapporte dans un inventaire de 1635 que certaines œuvres d'art ont disparu à la suite de la conquête du Palatinat par les troupes impériales et espagnoles :

3 Messinge Kunstbilder von ungever 12 oder 16 Pfundt metal

1 Messingbilt Ritter St. Georg

1 Bleyenes bilt, so hindter einer Felssn gestanden in der Schrotten $[\text { Khrotten? }]^{30}$

D'autres matériaux provenant de la grotte et de ses équipements hydrauliques ont également été décrits :

29. GLA KA, 391/14863, fol. 9-31.

30. Bayerisches Hauptstaatsarchiv [Archives de la Bavière], HR I Fasz. 165 n $^{\circ}$ 33/2, "Verzaichnus der paum so noch im Schlossgartten Heydelberg vorhanden " [Inventaire des arbres qui subsistent des jardins du château de Heidelberg]. Trad. fra : 3 figures en cuivre pesant environ 12 à 16 livres; 1 figure en cuivre du Chevalier de Saint-Georges ; 1 figure en plomb debout derrière un rocher dans la grotte ( ?). 
Vil Behrlemutter Muschelen, von rotten und weisen Corralen Zinckhen. Und ist sonsten daß Wasserwerckh, an ihm selber noch gangbar, und sein alle Instrument, so man uf dem / Tisch springen lest, noch verhanden. Auch ist noch eine grosse anzall berlemutter Muscheln bei der Hand, wie auch dreij Säckhe von guetten als rotten wie auch weissen Corallen zinckhen bei der Hand ${ }^{31}$.

Ainsi, en dehors de quelques récits de voyageurs qui mentionnent l'existence des jeux d'eaux et de l'entretien, et qui témoignent de l'intérêt que leur portait le prince-électeur à la fin du XVII ${ }^{\mathrm{e}}$ siècle, aucun autre document ancien ne mentionne la grotte ${ }^{32}$. La Brunnenstube ne peut donc pas être datée uniquement à partir des sources écrites. Mais l'analyse des données matérielles subsistantes permet une datation assez précise.

\section{L'extérieur}

Le portail d'entrée de la Brunnenstube est une construction semblable à celle l'escalier d'angle en colimaçon qui se trouve à proximité dans le mur est. Contrairement au portail de la grande Grotte ornée avec des éléments architecturaux à décor rustique animalier, l'entrée est située dans la paroi murale, discrètement rustiquée et simplement surmontée d'une architrave. Elle a des lignes sobres et régulières, avec un linteau droit et un fronton central. L'inscription sur la frise et la corniche de l'architrave peut être restituée : FRIDERICVS V COMES PALATINVS EL(ector) D(ux) $\mathrm{B}(\text { avariae })^{33}$.

Quant aux terrasses supérieures, elles avaient nécessairement dû être aménagées avec leurs canalisations et leurs regards de distribution des eaux avant la construction de la salle. C'est ce que confirme une livraison importante de sable en 1616 et le fait que le jardinier Peter Leonhardt avait déjà commencé à travailler en 1618. En effet, l’entrepreneur de la grotte a déjà

31. HR I Fasz. $165 n^{\circ}$ 33/2. Trad fra : Nombreuses coquilles de nacre, branches de corail rouge ou blanc. Le système hydraulique avec tous ses équipements est encore là, les jeux d'eau de la table fonctionnent encore. On trouve aussi un grand nombre de coquilles de nacre en stock, ainsi que trois sacs avec de belles branches de corail rouge ou blanc.

32. Gensichen, 2019, p. 137-138, n. 28 à 31.

33. La même inscription, avec la date " 1619 ", se trouve dans le parterre des muses sur la terrasse principale. Par contre, l'inscription sur le portail similaire de l'escalier en colimaçon à l'est du Brunnenstube ne peut plus être restituée. 
réalisé les quatre « Bronnenstuben » en 1619, comme l'indique un marché du 14 décembre $1619^{34}$.

D'ailleurs, l'inscription du portail ne mentionne que les titres de Frédéric de Comes palatinus, Elector et dux Bavariae, alors que l'inscription de la fontaine de Neptune le mentionne déjà comme Bohemiae rex. La Brunnenstube fut donc construite dans les années précédant le couronnement du roi de Bohême en novembre 1619, et même probablement peu après l'année 1616.

\section{L'intérieur}

La grande salle d'environ 4 mètres sur 4 mètres à trois niches est l'unique trace matérielle du dispositif hydraulique provenant des coteaux du Königstuhl pour alimenter l'ancien Hortus Palatinus. Les vestiges du décor mural ont donc une importance particulière. Les dommages causés au mur sud par l'effondrement décrit précédemment sont encore nettement visibles. La réparation des murs puis l'installation de dalles de pavement au $\mathrm{Xx}^{\mathrm{e}}$ siècle ont complètement détruit l'ancien sol, mais on peut supposer qu'il y avait un bassin au moins dans la plus grande des trois niches au mur sud vers la montagne.

Les vestiges sont incomplets et ne permettent pas de reconstituer l'ensemble du décor ancien. Mais tous les éléments caractéristiques d'un mur de grotte sont présents : la décoration des parois supérieures est soulignée par une étroite corniche sous laquelle des arcs en anse de panier décorent les murs est et ouest. Sur la corniche, on trouve des vestiges de fleurs à quatre pétales avec des coquilles, notamment des ormeaux ; il s'agit de variantes plus petites des splendides fleurs de coquillage de l'Architectura civilis de Furttenbach (Fig. XXIII, cahier couleur) ${ }^{35}$. La corniche et les parois supérieures étaient recouvertes de pseudo-pierres de tuf - un mortier fin imitant le tuf ${ }^{36}$ - qui sont encore partiellement conservées, en particulier sur

34. GLA KA, 204/517, livraison de sable au château de Heidelberg, 1616 ; GLA KA, 67/930, fol. 496r-498r, marché de jardinage pour Peter Leonhardt, pour tous les jardins du prince-électeur à Heidelberg, jusqu’à Noël 1618 ; fol. 544v-545v, marché pour la construction de la grotte par Peter Ancellin, 14 décembre 1619 (publié par Zangenmeister, 1886, p. 145-148.

35. Ormeau (Haliotis sp.), un gastéropode marin, souvent utilisé pour la décoration des grottes en raison de sa grande surface nacrée. Furttenbach, 1628, p. 47 et pl. n 20.

36. Böttcher, 2011, p. 9-12. 
la paroi au-dessus de l'entrée dans le mur nord. Les quelques gros vestiges conservés entre la voûte et les murs ressemblent aux décors dessinés par de Caus pour la petite Grotte, où les bordures de toutes les surfaces murales sont soulignées par de grandes pierres grossièrement taillées. Le mur au-dessus de l'entrée conserve l'unique trace d'un décor disposé sur l'axe central (Fig. XXIV, cahier couleur) : les fragments d'un masque au-dessus d'un motif central rappelant une fleur de $\mathrm{lys}^{37}$. Une corne d'abondance est conservée sur la droite, tandis qu'à gauche des traces d'une bordure correspondent à son pendant. La surface des formes en relief est finement marquée par la mise en place de différents types de coquilles alignées en rangs serrés. Des restes de stalactites sont également visibles.

Le masque est un bel élément sculpté, il se détache d'un bloc de grès. Les vestiges des coquilles et de pierre de tuf, qui ont souvent disparu, ont néanmoins laissé des empreintes visibles en surface ; certaines parties auraient pu être constituées de branches de corail. Un deuxième masque, dont il ne reste que l'arrière en stuc, se trouve en dessous, directement au-dessus de l'entrée. Sur le mur sud, au-dessus de l'arcade de la niche, un haut bloc de grès rectangulaire portait probablement un autre masque. On identifie aussi des fragments de divers matériaux, comme de l'ardoise couverte de fragments de verre verts incrustés dans les petites anfractuosités des arcs de voûte (Fig. XXV, cahier couleur). Le rapport de Johann Metzger de 1829 rappelle l'ancienne splendeur de l'aménagement de cette grotte :

Die Muschelarten, die sich daselbst befanden, waren: Heliotis marmorata, Pectunculus villosus, Avicula margaritifera, Cardium tuberculatum, Venus Chione; mehrere Cyprearten, Turbo argirostomus, Buccinum undatum, Mytilus edulis und mehrere andere. Ferner von Corallenarten: Matripora muricata, M. ramea, eine Art Antipetes und andere. ${ }^{38}$

La salle était donc ornée de moules, de pétoncles, de vénus, d'escargots de mer et plusieurs sortes de porcelaines, c'est-à-dire aussi de précieuses

37. Ce motif est utilisé par Salomon de Caus dans le parterre des Muses : De Caus, 1620, pl. 5. 38. Metzger J., 1829, p. 100. Trad. fra. : Les espèces de coquilles qui se trouvaient là étaient : Heliotis marmorata, Pectunculus villosus, Avicula margaritifera, Cardium tuberculatum, Venus Chione; plusieurs espèces de cypraeas (porcelaines), Turbo argirostomus, Buccinum undatum, Mytilus edulis et plusieurs autres. Également des espèces coralliennes : Matripora muricata, M. ramea, une espèce Antipetes et d'autres. 
espèces tropicales ${ }^{39}$. De plus, la pièce était revêtue de pierres taillées fixées avec des clous et probablement aussi avec des pierres semi-précieuses ${ }^{40}$. Vu de l'extérieur, le style classique et dépouillé du portail faisait un étonnant contraste avec l'intérieur scintillant de la Brunnenstube.

\section{Une grotte de Salomon de Caus?}

L'analyse de ces découvertes n'exclut donc pas une datation de la grotte remontant à l'origine de l'Hortus Palatinus ou peu de temps après. En effet, les matériaux encore présents dans la grotte ou signalés par Johann Metzger sont caractéristiques de cette époque. Par ailleurs, la forme structurée et régulière de la salle justifie d'une telle datation. Le répertoire des formes avec les volutes, les mascarons et les cornes d'abondance, l'organisation architecturale structurée, sont également compatibles avec les ouvrages réalisés par Salomon de Caus. Ainsi, le mascaron qu'il a gravé sur une fontaine dans un parterre à l'entrée d'un jardin ${ }^{41}$ a un décor semblable, orné de coquilles et de rocailles.

Les murs intérieurs de la Brunnenstube au nord, à l'est et à l'ouest conservent les restes d'une mosaïque compartimentée, avec une utilisation de somptueux bivalves et gastéropodes marins. Les gracieuses arcades en anses de panier avec leurs incrustations ornementales excluent qu'il y ait eu des niches plus grossières qui auraient imité les grottes naturelles. On peut émettre l'hypothèse que les délicates mosaïques de diverses coquilles et la structure très architecturée dominaient l'ensemble de cette pièce. Éventuellement, cette décoration contrastait avec une arcade plus grossière au sud. Le texte de Salomon de Caus sur les autres grottes permet de l'imaginer. Il décrit le système ornemental de la petite Grotte « en façon de mosaïque $»^{42}$, tandis que pour les voûtes de la grande Grotte, il donne deux descriptions différentes : " une partie, dont le vouste est faite de plusieurs

39. Il est possible que Salomon de Caus ait employé des coquilles exotiques comme il l'a fait pour les jardins de la Warande à Bruxelles, importées par le port de Rouen : De Jonge, 2000, p. 92, n. 23. 40. «mit Drath [sic !], Nägel und Speiß befestigt waren, verziert » [décorés et fixés avec du fil de fer, des clous et du mortier] : Metzger J., 1829, p. 100. On ne sait pas exactement si Metzger a vu ces espèces de coquillages ou s'il a utilisé une ancienne description inconnue aujourd'hui. Des petites pointes de fer sont conservées dans un endroit de la grande Grotte.

41. De Caus, 1620, pl. 16.

42. De Caus, 1620, n.p., Av Lectevr [n 26]. 
compartimens \& et figures de coquilles : L'autre partie est Rustique ${ }^{43}$. Sur le mur est, il y avait une petite surface avec du pseudo-tuf, comme dans la Brunnenstube. On ignore comment se faisait cette répartition ${ }^{44}$. Il ne reste plus rien des coquilles ou des rocailles qui ornent les arêtes des voûtes représentées sur la planche $\mathrm{XXII}{ }^{45}$.

D’une manière générale, il s'agit d'un schéma décoratif formellement moderne, fortement architecturé avec des éléments caractéristiques des grottes employant des matériaux exotiques, rares et coûteux. En l'état de nos connaissances, rien ne s'oppose à ce que quelques vestiges de l'époque de Salomon de Caus, ou immédiatement après, soient conservés dans la Brunnenstube. Le haut niveau de qualité de la décoration de cette petite salle est encore visible malgré son état fragmentaire. Il reste encore une part de sa splendeur perdue dans la partie centrale de la grotte.

La comparaison avec les décorations des grottes contemporaines confirme également la datation des vestiges du début du XvII ${ }^{\mathrm{e}}$ siècle. Le contraste entre la conception classique des murs d'entrée et l'intérieur en forme de grotte apparaît nettement dans la grotte de l'ancienne abbaye cistercienne 142 de Woburn, en Angleterre dans le Bedfordshire. Cette grotte a été aménagée vers 1630 avec des bandes de coquilles qui soulignent les arcades de la voûte ${ }^{46}$. Les parois compartimentées de la Brunnenstube peuvent aussi avoir ressemblé à celles de la grotte du château de la Bâtie d'Urfé (près de Lyon) avec des surfaces de petits galets de rivière, de rocailles et de coquilles. Le grand mascaron au-dessus de l'entrée de la Brunnenstube à Heidelberg représente un motif typique de l'époque, même dans (ou précisément à cause de) son état fragmenté, étrange, fantastique et grotesque, comparable à la tête de terme par Bernard Palissy, ou la tête du terme de la fontaine Doria Galleani à Gênes ${ }^{47}$.

43. De Caus, 1620, n.p., Av Lectevr [n 21$]$.

44. La combinaison des deux manières, l'art des formes raffinées et l'aspect naturel plus grossier, caractérise aussi les aménagements de Pratolino. Metzger W., 2013, p. 579.

45. De Caus, 1620, pl. 22.

46. La grotte de l'abbaye de Woburn est une grotte située à l'intérieur du bâtiment ; elle est en grande partie conservée dans son état d'origine : Duggan, 2003, p. 52-53 et fig. 4. En raison de similitudes stylistiques, le dessin est attribué à Isaac de Caus, un frère de Salomon, qui a continué à utiliser les dessins de son aîné. Morgan, 2007, p. 33-36 ; Duggan, 2009 ; Vérin 2017, p. 67 et note 9.

47. Bernard Palissy et son atelier, terme anthropomorphe entouré de moules pour la grotte des Tuileries, vers 1565-1570, moulage en plâtre d'après le moule original trouvé aux Tuileries au 
Il est peu probable qu'une salle aussi précieusement ornée ait servi uniquement de chambre hydraulique. Dans la Scenographia de Matthäus Merian, dans la vue de l'Hortus Palatinus depuis l'est et dans la peinture de Jacques Fouquier, les joueurs de palemail frappent leurs balles à cet endroit, comme Salomon de Caus l'avait prévu sur cette terrasse ${ }^{48}$. Dans la Brunnenstube, juste à côté de la piste de jeu, les joueurs pouvaient se rafraîchir après une partie acharnée. La fonction et les aménagements de la Brunnenstube, cette luxueuse chambre de collecte des eaux, située dans le mur de soutènement sud, sous les coteaux, était entièrement dédiée à l'eau. Au même niveau, plus à l'ouest, se trouvaient les bains où l'eau abondait avec la petite Grotte. Plus haut, dans l'angle sud-est du jardin, Salomon de Caus projeta une fontaine de Neptune dans ses gravures avec, au-dessus, la sculpture géante du Prince Électeur Frédéric V. Les dieux-fleuves de la région, le Rhin, le Main et le Neckar devaient être réunis en dessous. Cet agencement illustre la tradition littéraire de la seconde moitié du Xvi ${ }^{\mathrm{e}}$ siècle selon laquelle la cour de Heidelberg était à la fois un nouveau Parnasse et la montagne des Muses ${ }^{49}$. Car c'est le fils de Neptune, Pégase, qui fit couler de son sabot la source sacrée d'Hippocrène, et son cavalier Apollon le conduisit au Parnasse au pied duquel jaillit la fontaine de Castalie. Ici, au plus haut niveau du jardin et sous le regard de Neptune, dans la niche de la fontaine située directement sous la statue monumentale de Frédéric V, l'eau - l'élément vital le plus important, à côté du soleil apollonien - a été prélevée du Parnasse à Heidelberg et conduite à travers le haut mur de soutènement dans les salles des grottes et des bains. Elle y déployait sa force motrice pour la production des effets mécaniques et de la musique. La grande Grotte recevait son eau du réservoir protégé par Vénus. Plus petite, la Brunnenstube était le plus haut des quatre réservoirs de l'Hortus Palatinus et le plus proche des sources des coteaux. C'était donc le premier endroit à recevoir et à mettre en scène l'abondante eau pure de la montagne, ce qui explique pourquoi sa décoration était si précieuse. Ces eaux étaient une richesse offerte par la nature, à la fois fertile et terrible, entrầnant peu à peu la dégradation des ouvrages d'art du jardin maniériste et provoquant jusqu'à la disparition de la Brunnenstube elle-même.

XIX ${ }^{e}$ siècle, musée Carnavalet, Paris ; Viennet, 2010, p. 60-61. La glaçure typique de Palissy était certainement prévue pour cette pièce ; Hanke, 2008, p. 255, fig. 4 et 5.

48. de Caus 1620, n.p., Av Lectuvr [n 20].

49. Hubach, 2002. 


\section{Archives}

\section{Stadtarchiv Heidelberg (SA HD)}

Salomon De Caus: Hortus Palatinvs a Frederico Rege Boemiae Electore palatino Heidelbergae extrvctvs Architecto 1620 Francofurti apud Ioh: Theod: de Bry

\section{Generallandesarchiv Karlsruhe (GLA KA)}

- 67/930, fol. 199v-200v (Anstellungsvertrag De Caus, 14.7.1614); fol. 496r-498r, Anstellungsvertrag Peter Leonhardt, Hofgärtner für alle kurfürstlichen Gärten in Heidelberg „uff Weihnachten“ 1618 ; fol. 544v545v (Anstellungsvertrag des Grottenmeisters Peter Ancellin, 14.12.1619).

- 204/517 (Fronschuldigkeit der Zehnten zu Schriesheim und Kirchheim zur Führung von Sand zum Heidelberger Schlossgarten, 1616).

- 391/14863, fol. 9-31 (Bericht des Chausseeinspektors Funk, 15.8.1810). 424 Zug. 1998-40/200, Schreiben der Oberfinanzdirektion Karlsruhe an das Staatliche Hochbauamt Heidelberg, 08.06.1959.

\section{Bayerisches Hauptstaatsarchiv München}

Fürstensachen, Nr. 159b (Gehaltsliste der Heidelberger Bauadministration, 1616). Hofamtsregister I, Fasz. 165, Nr. 33/2 (P. Leonhards Verzeichnis der noch vorhandenen Bäum etc., 1635).

\section{Vermögen und Bau Baden-Württemberg, Amt Mannheim und Heidelberg}

Böttcher 2011

Silke Böttcher: Schloss Heidelberg, Hortus Palatinus, Terrassenmauer T, Muschelgrotte, Bestands- und Zustandsaufnahme, Restaurierungskonzept, Nov. 2011 (unveröfftl. Untersuchungsbericht i. A. v. Landesbetrieb Vermögen und Bau Baden-Württemberg, Amt Mannheim und Heidelberg).

Diener 2007

Andreas Diener : Befund-Dokumentation auf der Unteren Terrasse im Hortus Palatinus, 3.-10. September 2007, Institut für Europäische Kunstgeschichte, Zentrum für europäische Geschichts- und Kulturwissenschaften der Universität Heidelberg (unveröffentlichtes Manuskript, i. A. v. Landesbetrieb Vermögen und Bau Baden-Württemberg, Amt Mannheim und Heidelberg).

Gensichen 2010

Sigrid Gensichen : Die Quellen zum Heidelberger Schlossgarten 1614 bis 1960/61 : Die Mauern T und VR, Oktober 2010 (unveröffentlichtes Manuskript, i. A. v. Landesbetrieb Vermögen und Bau Baden-Württemberg, Amt Mannheim und Heidelberg)

Gensichen 2014

Sigrid Gensichen : Errichtung und Bauerhaltung der großen Grotte, ihres Nebenraumes und der Mauer VR im Heidelberger Schlossgarten (Hortus Palatinus), 1614 bis 2014 (Text und Zusammenstellung der beteiligten 
Gewerke). Dokumentation nach Abschluss der Maßnahmen, November 2014 (unveröffentlichtes Manuskript, i. A. v. Landesbetrieb Vermögen und Bau Baden-Württemberg, Amt Mannheim und Heidelberg)

\section{Sources imprimées}

De Caus Salomon, Hortus Palatinus a Frederico Rege Boemiae Electore palatino Heidelbergae extrvctvs Architecto, Francofurti apud Ioh : Theod : de Bry, 1620 [édition française : Paris, 1981 ; édition allemande : Neudruck, hg. v. Reinhard Zimmermann, Worms, 1980].

FurTtendach Joseph, Architectura civilis (1628). Architectura recreatinonis (1640). Architectura privata (1641). [Drei Bände in einem Band. Mit einer Vorbemerkung von Hans Foramitti, Hildesheim/u. a., 1971].

Galle Philipp, Semideorum marinorum amnicorumque sigillariae imagines perelegantes, Antwerpen, 1586, URL : http://mdz-nbn-resolving.de/ urn:nbn:de:bvb:12-bsb11346458-7.

Galle Philipp, Nimpharum oceanitidum, ephydridum potamidum, naiadum, in gratiam picturae studiosae iuventutis delineatae, scalptae, et editae a Philip Gallaeo lynadumque icones, Antwerpen, 1587.

MetzGer Johann, Beschreibung des Heidelberger Schlosses und Gartens. Nach gründlichen Untersuchungen und den vorzüglichsten Nachrichten bearbeitet von Johann Metzger, Universitätsgärtner und mehrever gelehrten Gesellschaften Mitglied. Mit 24 in Aquatinta, von C. Rohrdorf gestochenen Kupfertafeln, Heidelberg, 1829.

\section{Bibliographie}

BötTCHeR Silke, « Materialtechnische Befunde zur Brunnenstube», dans HaYton Nichola M.V., Hubach Hanns et Neumaier Marco (dir.), Churfürstlicher Hochzeitlicher HeimführungsTriumph. Inszenierung und Wirkung der Hochzeit Kurfürst Friedrichs V. mit Elisabeth Stuart (1613), verlag regionalkultur, UbstadtWeiher, 2019, p. 151-152.

Buchanan Iain, "The Collection of Niclaes Jongelinck: I. 'Bacchus and the Planets' by Jacques Jongelinck ", The Burlington Magazine, n 132, 1990, p. 102-113.

Buffa Géraud, Rostaing Amélie, Lurin Émmanuel, «Les merveilles hydrauliques des grottes et des jardins ", dans Lurin Émmanuel (dir.), Le Château-Neuf de Saint-Germain-en-Laye, Les Presses franciliennes, Saint Germain-en-Laye, 2010, p. 104-115.

De Jonge Krista, «Ein Netz von Grotten und Springbrunnen. Die "Warande” zu Brüssel um 1600 ", dans Härtuing Ursula (dir.), Gärten und Höfe der Rubenszeit im Spiegel der Malerfamilie Brueghel und der Künstler um Peter Paul Rubens 
(catalaogue d'exposition, Gustav-Lübcke-Museum Hamm/Landesmuseum Mainz), Hirmer, Münich, 2000, p. 89-105.

Devigne Marguerite, "Salomon de Caus ", Revue belge de philologie et d'histoire, $\mathrm{n}^{\circ} 15$, fasc. 2, 1936, p. 652-658.

Duggan Dianne, "Isaac de Caus, Nicholas Stone and the Woburn Abbey Grotto ", Apollo NF, n 158, 2003, p. 50-58.

Duggan Dianne, "Isaac de Caus. Surveyor, Grotto and Garden Designer ", Studies in the History of Gardens \& Designed Landscapes, vol. 29, n 3, 2009, p. $152-168$.

Gensichen Sigrid, "Flussgötter, Quellnymphen und eine Brunnenstube. Der Heidelberger Hortus Palatinus im Kontext der pfälzisch-englischen Hochzeit 1613 », dans Hayton Nichola M.V., Hubach Hanns et Neumaier Marco (dir.), Churfürstlicher Hochzeitlicher HeimführungsTriumph. Inszenierung und Wirkung der Hochzeit Kurfürst Friedrichs V. mit Elisabeth Stuart (1613), verlag regionalkultur, Ubstadt-Weiher 2019, p. 131-168.

Gensichen Sigrid, Metzger Wolfgang, "Johann Metzger als Gärtner des Heidelberger Schlossgartens ", dans Rink Claudia et Moritz Werner (dir.), Mit Spaten und Feder. Johann Metzger (1789-1852) - Landschaftsarchitekt, Botaniker und Gestalter des Heidelberger Schlossgartens, verlag regionalkultur, Ubstadt-Weier, 2008, p. 12-17.

Hanke Stephanie, Zwischen Fels und Wasser. Grottenanlagen des 16. und 17. Jahrhunderts in Genua, Rhema, Münster, 2008.

Hepp Frieder, LeIner Richard, Mach Rüdiger et al., Magische Maschinen. Salomon de Caus' Erfindungen für den Heidelberger Schlossgarten 1614-1619, Pollichia, Neustadt a. d. Weinstraße, 2008.

Hubach Hanns, "Parnassus Palatinus. Der Heidelberger Schloßberg als neuer Parnaß und Musenhort ", dans Gercke Hans, Der Berg (catalogue d'exposition, Kunstverein Heidelberg), Kehrer, Heidelberg, 2002, p. 84-101.

HuвACH Hanns, «Zur Zusammensetzung der kurpfälzischen Bauadministration in Heidelberg 1616. Marginale Quellenfunde zu Salomon de Caus und zum Hortus Palatinus ", dans Hepp Frieder, LeIner Richard, МaCH Rüdiger et al., Magische Maschinen. Salomon de Caus' Erfindungen für den Heidelberger Schlossgarten 1614-1619, Pollichia, Neustadt a. d. Weinstraße, 2008, p. 38-43. ICKowicz Pierre, " Nature et artifice : Grotte-image au château de Dieppe », dans Cuisenier Jean (dir.), Matière et figure, La Documentation française, Paris, 1991, p. 185-195.

Icкоwicz Pierre, «Une grotte artificielle du xvi ${ }^{\text {- }}$-xvii ${ }^{\text {e }}$ siècle au château de Dieppe, Seine-Maritime ", Monumental (Les chantiers de restauration du Centre des monuments nationaux), $\mathrm{n}^{\circ} 2,2014$, p. 80-81. 
Icкоwicz Pierre, "La grotte artificielle du château de Dieppe : une œuvre de Salomon de Caus?", Bulletin monumental, tome 174-4, 2017, p. 391-396.

Meijer Bert, "The Re-emergence of a Sculptor: Eight Lifesize Bronzes by Jacques Jonghelinck ", Oud Holland, n 93, 1979-2, p. 116-135, URL : https://doi. org/10.1163/187501779X00177.

Metzger Wolfgang, "Wasserkunst" und "Krottenwerk" - de Caus' Hortus Palatinus im 17. Jahrhundert ", dans Hepp Frieder, Leiner Richard, MaCH Rüdiger et al., Magische Maschinen. Salomon de Caus' Erfindungen für den Heidelberger Schlossgarten 1614-1619, Pollichia, Neustadt a. d. Weinstraße, 2008, p. 65-74.

Metzger Wolfgang, "The Perspective of the Prince. The Hortus Palatinus of Friedrich V and Elizabeth Stuart at Heidelberg ", dans Smart Sara et Wade Mara R. (dir.), The Palatine Wedding of 1613. Protestant Alliance and Court Festival, Harrassowitz, Wiesbaden, 2013, p. 567-596.

Morgan Luke, Nature as Model. Salomon de Caus and Early Seventeenth Landscape Design, University of Pennsylvania Press, Philadelphia, 2007.

Untermann Matthias, Gross Uwe, "Geheimgang und Kasematten. Archäologische Beobachtungen im Heidelberger Schlossgarten ", Archäologische Ausgrabungen in Baden-Württemberg, 2005, p. 261-264.

Vérin Hélène, «De princes en monarques : Salomon de Caus (1576-1626) et l'art des grottes, fontaines et jardins ", dans Medvedkova Olga (dir.), Les Européens : ces architectes qui ont bâti l'Europe (1450-1950), Peter Lang, Bruxelles, 2017, p. 65-85.

Viennet Christine, Bernard Palissy et ses suiveurs du XVI siècle à nos jours. Hymne à la nature, Faton, Dijon, 2010.

Zangenmeister Karl, "Ansichten des Heidelberger Schlosses bis 1764 ", Mitteilungen zur Geschichte des Heidelberger Schlosses, n 2-4, 1886, p. 35-160.

Zimmermann Reinhard, Hortus Palatinus. Die Entwürfe zum Heidelberger Schlossgarten von Salomon de Caus 1620, Werner, Worms, 1980.

\section{L'autrice}

Sigrid Gensichen a une formation en restauration, histoire de l'art et philosophie en Allemagne. Spécialisée dans la recherche et l'étude des sources d'archives des monuments et des jardins historiques, elle a collaboré au développement des bases de données pour inventorier les monuments historiques du Land de Mecklenburg-Poméranie-Occidentale et la collection lapidaire du château de Heidelberg. Elle est également spécialiste de l'art baroque du sud de l’Allemagne. Contact : sigrid.gensichen@gmx.de 